\title{
Diseases of the oral cavity in light of the newest epigenetic research: Possible implications for stomatology
}

\author{
Jadwiga Jośko-0chojska, ${ }^{1, A-D, F}$, Katarzyna Rygiel ${ }^{1, D, E, F}$, Lidia Postek-Stefańska ${ }^{2, B, C, F}$ \\ ${ }^{1}$ Department of Environmental Medicine and Epidemiology, Medical University of Silesia, Zabrze, Poland \\ ${ }^{2}$ Department of Pediatric Dentistry, Medical University of Silesia, Zabrze, Poland \\ A - research concept and design; $B$ - collection and/or assembly of data; $C$ - data analysis and interpretation; \\ $\mathrm{D}$ - writing the article; $\mathrm{E}$ - critical revision of the article; $\mathrm{F}$ - final approval of the article
}

Address for correspondence

Katarzyna Rygiel

E-mail: kasiaalpha@yahoo.co.uk

Funding sources

None declared

Conflict of interest

None declared

Received on April 7, 2017

Reviewed on May 9, 2017

Accepted on July 24, 2017

Published online on October 2, 2018
Cite as

Jośko-0chojska J, Rygiel K, Postek-Stefańska L. Diseases

of the oral cavity in light of the newest epigenetic research:

Possible implications for stomatology. Adv Clin Exp Med.

2019;28(3):397-406. doi:10.17219/acem/76060

DOI

10.17219/acem/76060

\section{Copyright}

Copyright by Author(s)

This is an article distributed under the terms of the

Creative Commons Attribution Non-Commercial License

(http://creativecommons.org/licenses/by-nc-nd/4.0/)

\begin{abstract}
Epigenetics is the study of inheritable changes in gene expression without changes in the underlying deoxyribonucleic acid (DNA) sequence. The main mechanisms of epigenetic regulation include DNA methylation, modifications in histones, and micro-ribonucleic acids (miRNA). Recent research evidence has shown that environmental and lifestyle factors dynamically interact with the genome, influencing epigenetic changes, from development to the later stages of life. This happens across a spectrum, from physiological to pathological conditions, such as genetic defects, developmental disorders, infectious or inflammatory processes, cancers, mental disorders, and substance abuse. Epigenetic studies have been conducted in various medical disciplines (e.g., oncology, internal medicine or psychiatry), adding valuable insight to standard medical approaches. However, in stomatology, epigenetic research is still in its infancy; thus, this review is aimed at presenting the role of epigenetic mechanisms in diseases of the oral cavity, including periodontal diseases, caries, developmental anomalies, and oral carcinoma. In addition, this paper reveals new insights into epigenetic biomarkers that can be helpful in the detection, early diagnosis, prognosis, and treatment of different oral diseases. Moreover, this review is focused on the possible clinical implications (diagnostic and therapeutic) of epigenetics, in the form of some noninvasive methods that can possibly be used in the future for the screening, work-up, outcome prediction and novel treatments of some dental diseases. Finally, this paper highlights that an epigenetic approach can be useful for designing novel interventions that will improve the management of oral malignancies or developmental abnormalities.
\end{abstract}

Key words: periodontal diseases, dental caries, epigenetics, developmental anomalies, oral squamous cell carcinoma 


\section{Introduction}

One of the biggest challenges in contemporary medicine is a deeper understanding of disease pathophysiology, since this knowledge can create novel diagnostic and therapeutic methods to potentially reduce the prevalence of various chronic diseases or developmental defects. Deoxyribonucleic acid (DNA) has so far been regarded as the only source of genetic information. If, however, genes which encode proteins are so important, why do they constitute only $2 \%$ of the human genome? In an attempt to explain this topic, recent research has indicated that in our cells, in addition to the genome (or the "first code"), there is also an epigenome (or the "second code"), which is a controlling superstructure of the "first code". ${ }^{1}$

Moreover, a modern approach to disease pathophysiology combines the current knowledge of genomic and environmental influences, and this connection is the main subject of a new medical science - epigenetics - that explores non-genomic inheritance. ${ }^{1}$

Since environmental and lifestyle factors can interact with the genome and can influence epigenetic changes, epigenetics can shed new light on the etiopathology of some genetic defects, developmental anomalies, mental or behavioral disorders, infectious or inflammatory changes, precancerous or cancerous conditions, and many other abnormalities. ${ }^{2}$ Due to the fact that epigenetic mechanisms are reversible, they can be affected by different factors, such as diet, medications, physical activity, mental or physical stress, traumatic events, environmental toxins, and addictive substances (e.g., nicotine and alcohol). ${ }^{2,3}$

Epigenetics is mostly a domain of genetics, biology, internal medicine, oncology, or psychiatry. In contrast, in stomatology, epigenetic research is still in its infancy. However, it should be noted that epigenetic mechanisms play an important role in gene expression during dental development (from the $6^{\text {th }}$ week of gestation to approx. the $20^{\text {th }}$ year of life), and that the potential impact of many environmental factors, over such a long period of time, can significantly disturb normal developmental processes and can influence the development of various oral diseases. Therefore, an understanding of epigenetic mechanisms is essential to the future implications for research and practice in the area of dentistry. 3,4

This review aims to describe the role of epigenetic mechanisms in diseases of the oral cavity, including periodontal diseases, dental caries, developmental anomalies, and oral carcinomas. Moreover, it presents some insight into epigenetic biomarkers, which can be beneficial in the early detection, diagnosis, prognosis, and treatment of different oral diseases. Finally, it highlights that epigenetics (in addition to current standard treatments) can be useful for designing novel interventions that will improve the management of some inflammatory processes, developmental abnormalities or malignancies in stomatology.

\section{Epigenetic mechanisms and their relevance to oral diseases}

Epigenetic mechanisms are "in charge" of transcriptional control (which regulates gene expression), and their interactions modify the structure and function of chromatin. Epigenetic modifications occur across the lifespan of the individual (from intrauterine environment to advanced age), and by altering gene expression patterns, they contribute to various cellular phenotypes. ${ }^{4-6}$ The key epigenetic mechanisms - including DNA methylation, histone modification and non-coding RNAs - and their relevance to oral diseases are briefly presented below.

\section{DNA methylation}

The process of DNA methylation, which represents the most characterized type of chromatin modification, involves the transfer of a methyl group ( $\mathrm{CH} 3$ ) from $\mathrm{S}$ adenosyl methionine (SAM) to cytosines present in cytosine-phosphate-guanine (CpG) dinucleotides of the DNA chain. The CpG sequences that are located throughout the genome are usually highly methylated and germlinespecific. The CpG methylation results in transcriptional repression. ${ }^{4,5}$ Abnormal methylation states can therefore lead to disease development. For instance, hypomethylation changes in DNA are related to chromosome instability that may lead to cancer. Based on recent studies in stomatology, DNA methylation patterns can be changed by inflammatory processes. Furthermore, alterations in DNA methylation patterns and cytokine gene expression can be seen in chronic periodontitis. Also, methylation patterns can differ between healthy and inflamed dental pulp. ${ }^{4,5}$

\section{Histone modifications}

A basic unit of chromatin, the nucleosome, consists of a DNA segment and 8 core histones. Such an octamer has 2 copies of $\mathrm{H} 2 \mathrm{~A}, \mathrm{H} 2 \mathrm{~B}, \mathrm{H} 3$, and $\mathrm{H} 4$, and creates a rigid structure to chromatin. During post-translational modification of the core histones, chromatin can be condensed or relaxed. This is a key epigenetic mechanism that regulates gene transcription. The modification of histones predominantly occurs at the N-terminal tails of the protein. Acetylation of the core histones leads to an "open" chromatin structure that enables transcription (e.g., the acetylated $\mathrm{N}$-termini promote a more relaxed conformation of the chromatin, which permits the recruitment of the basic transcription factors). In contrast, histone deacetylases remove the acetyl groups, so that the chromatin become more condensed (which represses gene transcription). Histone methylation can cause either an activated or a repressed chromatin state. ${ }^{4,5}$ 
Recent studies have reported that histone modifications may induce differentiation and mineralization in dental pulp stem cells. Histone acetylation and deacetylation play a crucial role in the regulation of gene expression. This, in turn, can promote pulp repair and regeneration, and thus, may be useful in restorative stomatology. ${ }^{4}$

\section{Noncoding RNAs}

Noncoding RNAs are RNA molecules that do not encode for protein. They include transfer RNAs (tRNAs), ribosomal RNAs (rRNAs), microRNAs (miRNAs), and shortinterfering RNAs (siRNAs). Both miRNAs and siRNAs can regulate gene expression without altering the DNA sequence. ${ }^{4}$ It has been reported that noncoding RNAs are involved in oral diseases such as oral cancer. Also, it has been demonstrated that miRNAs play essential roles in odontoblast differentiation. ${ }^{4,5}$

\section{Periodontal diseases}

Periodontal diseases are pathological processes involving the periodontium (including the gums [gingiva], the alveolar bone [alveolar process], the dental cementum, and the periodontal ligament). They represent a significant public health problem worldwide and affect up to $70 \%$ of the population, regardless of gender or age. The main causes of periodontal diseases include poor oral hygiene, bruxism, malocclusion, and some general medical diseases, as well as improper fillings and prosthetic appliances. Recently, it has been considered that the epigenetic mechanisms contribute to the development of periodontal diseases, since they often occur during inflammation, are localized (e.g., on the border of the biofilm of the gums around the teeth) and differ depending on the location. ${ }^{7}$

\section{Chronic periodontitis}

Chronic periodontitis is an inflammatory disease affecting the tooth support structures. It is usually caused by interactions between periodontal pathogens and an immunological response of the host. Epigenetic mechanisms might contribute to the development of chronic periodontitis (Table 1), by influencing the expression of the genes participating in the immunological and inflammatory responses. ${ }^{8}$

In particular, according to the study by de Faria Amormino et al., it was shown that the toll-like receptors (TLR) play an important role in the response to bacterial infections. These receptors are mostly located in the areas that are potential "gates" for infection. ${ }^{9}$

In addition, the authors of the study compared the methylation status and the expression of the TLR2 gene in samples of gum tissue derived from patients with chronic periodontitis (study group) and from healthy individuals (control group). The results showed that in patients with periodontitis, there is hypermethylation and low expression of the TLR2 gene, comparing to the control group. In addition, a correlation was found between the methylation level and the degree of inflammation (e.g., less vs more advanced). ${ }^{9}$

According to some earlier studies, a decreased expression of E-cadherin is characteristic of certain carcinomas, including breast, lung, prostate, stomach, and colon cancers. ${ }^{10}$ Likewise, an altered E-cadherin expression plays a role in the progression of chronic periodontitis. ${ }^{11}$ In addition, translational research studies conducted by Loo et al. explored some epigenetic changes in E-cadherin and cyclooxygenase 2 (COX-2) that occur simultaneously in cancer and in chronic periodontitis. In particular, it was found that in the patients with breast cancer and in the ones with chronic periodontitis, both E-cadherin and COX-2 were hypermethylated, in contrast to the control group. The authors emphasize that chronic periodontitis can, to some degree, be associated with hypermethylation of DNA, which is related to cancer risk factors. In addition, they believe that further studies on similar epigenetic changes might be very useful in diagnosing and treating chronic periodontitis. ${ }^{12}$

Similarly, the results of another study have revealed that an increase of acetylation in the histone $\mathrm{H} 3 \mathrm{~K} 9$ and in the promotor $\mathrm{CBP} / \mathrm{p} 300$, and a decrease in the activity of histone deacetylase caused an increased expression of proinflammatory cytokines (such as IL-1, IL-2, IL-8, and IL-12), and the development of chronic periodontitis. ${ }^{13}$ In some other studies, it was also found that in chronic periodontitis, hypermethylation of the promoter of the prostaglandin-endoperoxide synthase 2 (PTGS2) resulted in reduced COX-2 expression. ${ }^{14}$ In this way, some epigenetic biomarkers relevant to hypermethylation or hypomethylation have been suggested as being helpful for a better understanding of the pathophysiology of chronic periodontitis. They include: 1. hypermethylation and decreased expression of the tumor necrosis factor (TNF- $\alpha$ ) and cyclooxygenase 2 (COX-2) and 2. hypomethylation and increased expression of interferon $\gamma($ IFN- $\gamma$ ), transcriptional factors (e.g., nuclear factor $\kappa \mathrm{B}[\mathrm{NF}-\mathrm{k} \mathrm{B}]$ ), and signal transducer and activator of transcription (STAT) proteins. ${ }^{15,16}$

\section{Aggressive periodontitis}

Aggressive periodontitis, including localized aggressive periodontitis (LAP) and generalized aggressive periodontitis (GAP), is a type of periodontal disease with some specific features (Table 1). Although it is much more rare than chronic periodontitis, it usually affects younger patients, who are otherwise in good health. In contrast to chronic periodontitis, the main characteristics of aggressive periodontitis (both LAP and GAP) include rapid bone destruction, a discrepancy between the amounts of microbial deposits and the severity of the periodontal tissue destruction, 
Table 1. Epigenetic mechanisms in common diseases of the oral cavity and their potential clinical implications: emerging epigenetic biomarkers for prediction or diagnosis

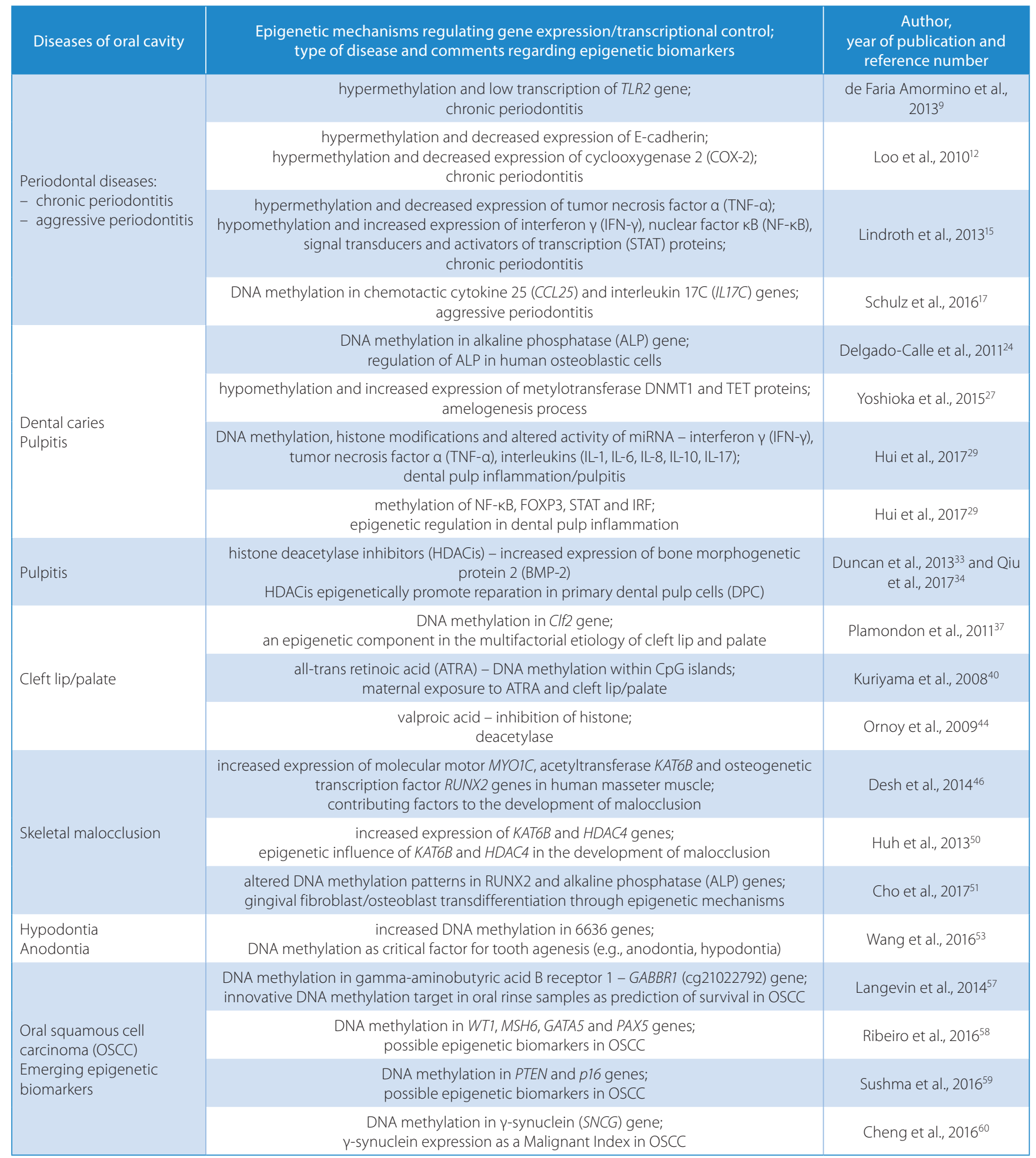

impaired neutrophil functions, abnormal chemotaxis and phagocytosis processes, hyperresponsive macrophage phenotypes (e.g., elevated levels of prostaglandin E2 [PGE2] and interleukin $1 \beta$ [IL-1 $\beta]$ ), as well as the typical involvement of a certain tooth (e.g., numbers $1-2$ and $6-7) .{ }^{17}$ Epigenetic modifications influence the immunological response that plays a key role in aggressive periodontitis. In a study of patients with acute periodontitis, Schulz et al., for the first time, examined the methylation of CpG in 22 inflammatory gene candidates (ATF2, CCL25, CXCL14, CXCL3, CXCL5, CXCL6, FADD, GATA3, IL10RA, IL12A, IL12B, IL13, IL13RA1, IL15, IL17C, IL17RA, IL4R, IL6R, IL-6, $I L 7, I N H A$, and $T Y K 2)$. They found that the methylation of CpG chemotactic cytokine 25 (CCL25) and interleukin 
17C (IL17C) was substantially lower than healthy periodontal tissues. Therefore, the decrease of CpG methylation probably accompanies an increase of expression of these genes. That, in turn, can lead to the increased availability of CCL25 (which is relevant to T-cell development) and IL17C (which regulates innate epithelial immune responses), causing pro-inflammatory reactions and contributing to a loss of attachment of the tooth in the alveolar fossa. Such epigenetic modifications can be triggered by many different external and internal factors (e.g., bacteria and demographic or socio-economic factors). ${ }^{17}$

\section{Dental caries}

Dental caries is a localized destruction of the tooth surface, initiated by decalcification of the enamel and followed by enzymatic lysis of organic structures, leading to cavity formation. If this condition is left unchecked and untreated, the cavity can penetrate the enamel and dentin, and then extend to the pulp. ${ }^{18}$ Tooth formation begins around the $34^{\text {th }}$ day of fetal life, and at this moment the process of amelogenesis (enamel formation) also begins. Amelogenesis is influenced by several internal (e.g., fever, hypoxia, lack of nutrients, and toxins) and external factors (e.g., antibiotics, environmental pollution and socio-economic status). ${ }^{18}$ Interestingly, some additional, novel factors, such as the impact of traumatic experiences of a pregnant mother on the development of the fetus, and later on, the child's dental structures, including early childhood caries (ECC), have been proposed. ${ }^{19}$

Furthermore, based on a study of 314 pairs of twins, aged from 1.5 to 8 years, it was found that if a pregnant mother suffers from depression, then the risk of ECC in her child increases. ${ }^{20}$ In addition, in a study by Hughes et al., in which 1,200 pairs of twins were examined using modern molecular methods, it was determined that epigenetic and environmental factors influenced tooth development and oral health. In particular, it was confirmed that genetic factors strongly influenced variation in the timing of primary tooth emergence. Also, ongoing follow-up clinical examinations of the twins are being conducted to examine whether those who become colonized earlier with decayforming bacteria will develop dental decay at an earlier age. In this way, a comparison within and between monozygotic and dizygotic twin pairs will help to explain the interactions between genetic, epigenetic and environmental factors, and their impact on oral health and disease. ${ }^{21}$

It should be highlighted that the concept of induced pluripotency (an "instrument" to obtain patient-specific stem cells) may provide some insight into the inter-relationships between transcription factors and chromatin structure and dynamics, which could be useful in stomatology. In particular, during cell differentiation, methylation of DNA might be the last step in the direction toward stabilization of cellular "destiny" (cellular reprogramming). ${ }^{22}$
This process is catalyzed by DNA methyltransferases (DNMTs) DNMT1 and DNMT3. DNMT1 is responsible for maintaining methylation in de novo formatted DNA strands, post replication, while DNMT3a and DNMT3b participate in de novo methylation during the embryonal processes of development and cell differentiation. ${ }^{23}$

DNA methylation contributes to the regulation of the functions of the osteoblasts, osteocytes, osteocalcin, and sclerostin. Moreover, DNA methylation plays a crucial role in controlling the expression of the alkaline phosphatase (ALP) gene, which regulates the transition of osteoblasts into osteocytes. ${ }^{24,25}$ Recent studies have revealed that the ten-eleven translocation (TET) protein family (TET1, TET2, and TET3) plays a main role in the demethylation of DNA. In particular, they interact with proteins of complexes participating in histone modifications, and by influencing their activity and ability to bind to chromatin, they can change the methylation profile or histone acetylation. It should be noted that a decreased expression of the TET genes is related with the development and progression of different types of malignancies, and that an increased expression of the TET genes is related with the amelogenesis process. ${ }^{26,27}$ In addition, it has been revealed that the methylation of cytosine correlates with enamel development, especially during early developmental stages (e.g., the expression levels of methyltransferase DNMT1 during the early developmental stages were almost 3 times higher than those during the late stages). A similar correlation was found in regard to the expression levels of the TET protein family (e.g., the levels of expression of TeT1 were 3 times higher in the early developmental stages than in the late stages). Since the DNMT methyltransferases and the TET protein family appear to be the main factors for epigenetic reprogramming (Table 1), it is highly probable that the dynamic changes of cytosine methylation can be essential in the regulation of amelogenesis. ${ }^{27}$

In their studies on the correlations between epigenetic changes and the development of dental caries in children, Fernando et al. examined 3,000 families (from 2006 to 2011). In each of these families, the periods of pregnancy, childhood and adulthood were assessed and for the epigenetic tests, saliva samples were collected. However, until now, only pilot studies have been conducted, and due to the small sample sizes, it was impossible to draw meaningful conclusions. However, there is hope that in the future these pioneering studies on epigenetic variability in dental caries in children may provide some evidence of associations between epigenetic variability and social or environmental factors causing caries in the pediatric population. ${ }^{28}$

\section{Pulpitis}

Pulpitis is a complication of untreated dental caries that develops due to the harmful action of some strains of bacteria causing decay, as well as to trauma or other adverse 
factors. The inflammatory reaction of pulp is regulated by epigenetic modifications, in which environmental factors play an enormous role, as proinflammatory triggers. According to some recent research in stomatology, epigenetic changes (e.g., DNA methylation) can occur due to environmental changes, both external and internal, including inflammatory lesions, dysbiosis, immune system compromise, or abnormalities in cytokine regulation. ${ }^{4,15}$

According to the studies by Hui et al., it was determined that the epigenetic regulation of dental pulp inflammation occurs via the processes of DNA methylation, histone modification and changed activity of miRNA. At these 3 levels, the dynamic modulations of interferon $\gamma($ IFN- $\gamma$ ), TNF- $\alpha$ and a number of interleukins (e.g., IL-1, IL-6, IL-8, IL-10, and IL-17) play a main role in pulp inflammation. ${ }^{29,30}$

It should be noted that over the last 10 years, tissue engineering has gained a lot of attention, since it can offer an innovative approach to dental pulp regeneration, after damage due to an inflammation or other types of injury. These innovative strategies, aimed at regaining the tissue and restoring its function, are related to de novo dental pulp regeneration (e.g., filling the canal with vital tissues rather than filling it with artificial materials). In particular, in the case of pulp regeneration, the goal is to reestablish pulp and dentin tissues in the canal space. This includes revitalization or revascularization. Although this is difficult to achieve, some novel approaches have been proposed, such as cell-based (e.g., transplanting exogenous cells into the host) and non-cell-based revitalization procedures. However, there are still some unresolved issues that have to be investigated in research studies prior to the possible introduction of these techniques into dental practice. ${ }^{31,32}$

Current research has revealed that histone modification might induce the differentiation and regeneration of stem cells of the dental pulp via hypoacetylation, exerted enzymatically by the histone deacetylase (HDAC). The HDAC inhibitors (HDACIs) inhibit the activity of HDAC and alter the level of histone acetylation (Table 1). This may represent a potential new approach to the treatment of many dental diseases, (e.g., via promoting reparative processes in primary dental pulp cells). ${ }^{33}$ Furthermore, in recent neuropsychopharmacological studies, 2 inhibitors of the histone deacetylase - valproic acid (VPA) and trichostatin A (TSA) - were examined, focusing on their influence on inducing the reparative responses in stem cell cultures of primary dental pulp cells (DPC).$^{34}$ For instance, it was found that the administration of HDACIs stimulates, to a large degree, osteopontine and the expression of a growth factor - bone morphogenetic protein 2 (BMP-2). In addition, the HDACIs also promote differentiation of the pulp's stem cells without cytotoxic effects. These new data underscore the potential related to the possible use of low HDACI concentrations in the treatment of pulpitis. Moreover, it was found that HDACIs might be used as potentially new medications in monotherapy, or in combination with other pharmacological agents. ${ }^{34}$
It should be underscored that epigenetic mechanisms such as the acetylation and deacetylation of histones play a key role in the regulation of gene expression and might promote reparative changes of the pulp, offering the possibility of an innovative dental therapy in the future. ${ }^{35}$

\section{Birth defects}

\section{Cleft lip and/or palate}

Cleft lip and cleft palate (or facial cleft) is a group of conditions including cleft lip, cleft palate and both together. A cleft lip is an opening in the upper lip, extending into the nose, and a cleft palate is a condition where the roof of the mouth has an opening into the nose. Cleft lip and palate are birth defects that are caused by the improper joining of the tissues of the face during development. These defects can cause feeding difficulties, speech abnormalities, hearing impairment, and frequent ear infections. Cleft lip and/or palate, is one of the most prevalent birth defects in the human population (e.g., it occurs in approx. 1 per 1,000 births in the developed world and in 2-3 per 1,000 births in Poland). ${ }^{36}$

An animal model of human cleft lip and palate are the A/WySn mouse strain, in which the cleft occurs in $20 \%$ of the population. Plamondon et al. have found that in mice, epigenetic modifications in the form of methylation in the Clf2 gene contribute to the development of the cleft. ${ }^{37}$ In humans, genetic (e.g., maternal diseases) and environmental factors (e.g., maternal nutritional habits and substance use or dependence disorders, such as tobacco smoking, alcohol, addictive medications, or illegal drug use) are responsible for the development of this defect. These environmental factors, predominantly including tobacco smoking, influence the gene expression.

Based on a meta-analysis by Little et al. that included data from 32 case-control and cohort studies, it was reported that there is a statistically significant correlation between maternal tobacco smoking and cleft lip, with or without cleft palate, and also between maternal tobacco smoking and cleft palate. The authors suggest that this evidence is so strong that it can support anti-nicotine campaigns. ${ }^{38}$ On the other hand, according to the results of a study by Grosen et al. that examined a population of twins with cleft lip/palate from the Danish Twin Registry (including 9,146 people who were born in the years 1936-2004), such a correlation was not determined. ${ }^{39}$ Furthermore, there is growing evidence indicating that epigenetic processes play a role in abnormal craniofacial formation. For instance, in 2008, Kuriyama et al. showed that in newborn mice, methylation of DNA - in specific dinucleotide sequences of $\mathrm{CpG}$ - induced the formation of cleft palate (Table 1), when their pregnant mothers were given a preparation of all-trans retinoic acid (ATRA). ${ }^{40}$ Also, it should be noted that epigenetic mechanisms are 
related not only to the changes of DNA and histones, but also occur at the level of microRNA (miRNA), which participates in the regulation of $30 \%$ of human genes, contributing to normal cell development. Recent studies have shown that miRNA regulates the processes of proliferation, differentiation and apoptosis of cells which are necessary to normal embryonic development. ${ }^{41}$ Moreover, it has been shown that in animal models, during the development of maxillary and facial tissues, different types of miRNA play a key role in ontogenesis (e.g., regulating a protein cytoskeleton, influencing growth factors, and modulating signal transduction and transcriptional factors). ${ }^{42,43}$ In addition, the influence of valproic acid used by pregnant mothers on the development of cleft palate in their children was examined by Ornoy (Table 1). The results of this study suggest that valproic acid (which inhibits the histone deacetylase) causes changes in gene expression and contributes to the development of this defect. ${ }^{44}$

\section{Skeletal malocclusion}

A skeletal malocclusion is a misalignment or incorrect relationship between the teeth of the 2 dental arches when they approach each other as the jaws close. Malocclusion is classified as follows:

- class I, neutrocclusion, in which the molar relationship of the occlusion is normal (e.g., for the maxillary $1^{\text {st }}$ molar), though the other teeth have some abnormalities (e.g., spacing, crowding, and over- or under-eruption);

- class II, distocclusion (retrognathism or overbite), where the mesiobuccal cusp of the upper $1^{\text {st }}$ molar is not aligned with the mesiobuccal groove of the lower $1^{\text {st }}$ molar (it is anterior to it); and

- class III, mesiocclusion (prognathism, anterior crossbite or underbite), in which the upper molars are not placed in the mesiobuccal groove, but posteriorly to it (the mesiobuccal cusp of the maxillary $1^{\text {st }}$ molar lies posteriorly to the mesiobuccal groove of the mandibular $1^{\text {st }}$ molar) and the lower front teeth are usually more prominent than the upper front teeth (e.g., a large mandible or a short maxillary bone).

The skeletal muscles that are involved in movements of the jaws are very flexible, and able to react or adapt to different physiological stimuli, via contraction or release. According to the pioneering studies by Pandorf et al. on the epigenetic mechanisms affecting these skeletal muscle fibers, it was revealed that histone modifications at the myosin heavy chain (MHC) genes occur in the locus of these genes (e.g., in response to muscle unloading) ${ }^{45}$ It should be highlighted that the main transcription factor regulating osteogenetic processes is the Runt-related transcription factor 2 (RUNX2), and its expression in osteoblasts and osteoprogenitor cells is crucial for these processes. In particular, in patients who underwent a surgical treatment of class II or class III malocclusion in their masseter muscle, the expression of the MYO1C gene (which encodes myosin 1C) and of acetyltransferase KAT6B (which activates RUNX2) were examined (Table 1). The findings of this study indicated that the abovementioned epigenetic modifications contributed to the development of malocclusion. ${ }^{46}$ Furthermore, it was determined that there were highly significant associations between MYO1C and the KAT6B expressions. Therefore, the change in expression of the myosin genes confirms that the differences in fiber type in the masseter muscle are important contributors to osteogenesis and to the development of malocclusion. ${ }^{47}$ Over the last 10 years, research related to epigenetic control of skeletal muscle fiber types and osteogenesis has rapidly emerged. ${ }^{48}$ These epigenetic mechanisms often include acetylation of lysine residues in the chromatin through acetyltransferases (KAT) and deacetylases (HDAC) of the histones. ${ }^{49}$ In a study by Huh et al., conducted among patients undergoing surgical procedures for malocclusion, biopsies of the masseter muscle were obtained, and myosin genetic variability (contributing to the development of class III malocclusion) was examined. In particular, 2 functionally connected enzymes - KAT and HDAC - were compared, to determine their correlations with the muscular-skeletal system during the development of malocclusion (Table 1). The data from this study support the results of some other published reports related to the epigenetic regulation of muscle and bone growth. In particular, it was shown that the expression of the $K A T 6 B$ and $H D A C 4$ genes was a few times higher in the masseter muscles of patients with a "deep bite" than in the ones with an "open bite." In addition, in patients with class III malocclusion, the expression of these genes was significantly higher than in patients with class II malocclusion. Therefore, the authors suggest that epigenetic regulation through coordination of the actions of both KAT6B and HDAC4 can be essential for the entire motor complex of mastication, during the development of skeletal malocclusion. ${ }^{50}$

One of the unresolved issues in stomatology is alveolar bone resorption, due to periodontal diseases, inflammatory processes, or traumatic injuries. To address this problem, Cho et al. analyzed the osteogenic potential of human gingival fibroblasts (HGFs) via a direct transdifferentiation (stem cell reprogramming and differentiation, not only to tissues of origin, but also to other tissues) from HGFs to functional osteoblasts, through epigenetic modification and osteogenic signaling, with bone morphogenetic protein 2 (BMP2). It turned out that HGF treatment with 5-aza-2'-deoxycytidine (5-aza-dC) induced demethylation in the hypermethylated CpG islands of the osteogenic lineage marker genes RUNX2 and ALP. Furthermore, subsequent BMP2 treatment drove the fibroblasts to osteoblast lineage.

The osteoblastic alterations, mediated by epigenetic modifications, demonstrated the changed methylation patterns in the RUNX2 and ALP promoter regions, as well as their effect on gene expression (Table 1). Based on these 
findings, the authors concluded that the epigenetic modification permits the direct programming of HGFs into functional osteoblasts, indicating that this approach could initiate a potential new therapeutic direction in alveolar bone regeneration. ${ }^{51}$

\section{Anomalies of dental development}

Developmental dental anomalies represent marked deviations from the normal number, shape, size, and degree of tooth development. Both local and systemic factors can contribute to these abnormalities, and can be inflicted before or after birth. Aberrations in the normal number of teeth include hyperdontia (supernumerary or excess teeth), hypodontia (a congenital lack of 1 or more teeth or missing teeth) and oligodontia (a developmental absence of 6 or more teeth, excluding the $3^{\text {rd }}$ molars). Anomalies in the shape of teeth include microdontia (teeth that are smaller in size) and macrodontia (teeth that are larger in size than normal). In addition, various anomalies in shape can be present (e.g., dens invaginatus, talon cusp, dens evaginatus, gemination, fusion, root dilacerations, taurodontism, and concrescence). Such anomalies, in addition to their appearance, often create different dental problems, as well as difficulties during dental treatment. ${ }^{52}$ Hypodontia is caused by complex interrelations between genetic, epigenetic and environmental factors during dental development. In a pilot study that examined 6,636 human genes, conducted by Wang et al., it was found that there are significant differences in the level of genome methylation between people with hypodontia, in whom methylation increased, and the control group (people without hypodontia) (Table 1). Since the DNA samples in this study were only collected from the oral epithelial cells, the authors suggest that in the future additional studies focused on some other types of cells participating in odontogenesis would be merited. Furthermore, a larger sample size is needed to explore in depth the epigenetic mechanisms related to the development of hypodontia. Although the study by Wang et al. was only a pilot study, it highlighted the key role of DNA methylation in hypodontia. ${ }^{53}$ Similarly, studies on monozygotic pairs of twin, in which there were differences in the number of absent or additional teeth, also confirmed the impact of epigenetic modifications. ${ }^{54-56}$

\section{Oral cavity carcinomas}

In general, the prevalence of oral cavity carcinomas in children is much lower than in adults, and the majority of them develop in the oral mucosa (about 70\%) (e.g., on the tongue), in the maxillary and mandibular bones (very rarely), in odontogenic tissues (about 25\%), and in the salivary glands (about 5\%). In general, the prevalence of oral cavity carcinomas increases with age and men are more predisposed to them.

\section{Oral squamous cell carcinoma}

Oral squamous cell carcinoma (OSCC) is the most common malignant epithelial neoplasm of the oral cavity. It is often characterized by a heterogeneous clinical picture and an aggressive course. For these reasons, an early diagnosis and effective therapy are essential, including the emerging epigenetic approaches on top of the standard medical care (Table 1).

In their study, Langevin et al. identified in oral rinse samples of patients with OSCC new prognostic epigenetic biomarkers which can predict overall survival in OSCC. In particular, they identified 7 novel DNA methylation loci, 1 of which was validated using a custom pyrosequencing assay. The authors found that DNA methylation occurs in the gene encoding for gamma-aminobutyric acid $B$ receptor 1 (GABBR1) (cg21022792). Hopefully, in the future, the application of this noninvasive test can help predict the survival of patients with OSCC. ${ }^{57}$ Furthermore, in a recent study by Ribeiro et al., some additional epigenetic biomarkers for the potential prediction of survival in patients with OSCC were indicated. For instance, it was found that the methylation of the WT1 gene promoter is related to a better survival outcome in patients with OSCC. In contrast, the methylation of MSH6 and the GATA5 gene promoter is related with a worse survival outcome in OSCC patients (Table 1). In addition, the authors have shown that the methylation of the PAX5 gene promoter is linked with carcinoma of the tongue. ${ }^{58}$

According to a study on the South Indian population, it has been reported that some other epigenetic changes might contribute to the development of OSCC. In particular, it has been determined that the phosphatase and tensin homolog (PTEN) and p16INK4a (p16) genes are tumor suppressor genes associated with epigenetic alterations. In this study, the authors found that the low level of expression of PTEN and $p 16$ genes due to DNA methylation might contribute to the development of carcinoma, and thus, it can be useful in the prognosis of OSCC (Table 1). Moreover, the authors suggested that epigenetic changes in these genes might represent a valuable biomarker for the early detection of OSCC. ${ }^{59}$

Similarly, the expression of $\gamma$-synuclein (SNCG) is related to the development of various carcinomas, including OSCC (Table 1). In their study, Cheng et al. examined a correlation between DNA methylation in the SNCG gene and the development of OSCC, including the clinical symptoms of this malignancy. It was found that positive SNCG expression in patients with OSCC significantly correlated with cancer staging and lymph node metastasis. However, SNCG methylation did not correlate with its genetic expression or clinical-pathological variables in OSCC tissues. In addition, the authors suggested that DNA methylation in SNCG might cause progression in OSCC. ${ }^{60}$ The results of a meta-analysis by Singh et al., including studies published from 2000 to 2015, have revealed that the research 
on epigenetic mechanisms can provide useful knowledge on novel biomarkers for early diagnosis, prognosis and treatment of oral cavity carcinomas or some other oral lesions. $^{2}$

\section{The role of epigenetics in future research and implications for dental practice}

Future research exploring the role of epigenetics in oral diseases will broaden our knowledge of how epigenetic patterns affect the phenotypical expression of diseases such as periodontitis, dental caries, congenital orofacial malformations, and oral cancer. ${ }^{3}$

In particular, this research will help us to understand how the "interplay" between genes and the oral microbiome or local biofilm can affect epigenetic mechanisms (in the periodontal tissues and oral mucosa), in order to modify the inflammatory or immune responses. Moreover, the relevant epigenetic biomarkers need to be evaluated in preclinical and clinical studies. ${ }^{4}$

Furthermore, epigenetic remodeling of cells (e.g., dental pulp) to a pluripotent state creates the potential for epigenetic reprogramming that can possibly be useful in healing local tissue injuries. Unquestionably, future studies are necessary to address these issues and to explore the impact of epigenetics on dental health and diseases. This, in turn, will allow the development of innovative, safe and effective therapies, so that dental practice can benefit from new opportunities for the diagnosis, treatment and prevention of oral diseases that are difficult to manage. ${ }^{5}$ Furthermore, there is a chance to develop an epigenetic profile of an individual patient in order to deliver personalized dental care. Finally, the possibility of screening for potential oral health problems (starting from early childhood) might prevent diseases, attenuate their course, or improve the patient's functional level and quality of life. ${ }^{6}$

\section{Conclusions}

The field of epigenetics is rapidly developing and there is emerging evidence that environment and lifestyle can interact with the genome to influence epigenetic changes. Epigenetics plays an important role in gene regulation via the main mechanisms, such as DNA methylation, histone modifications and non-coding RNAs. These mechanisms affect gene expression. Exogenous factors (e.g., diet, physical activity, medications, toxins, psychophysical stress, traumatic experiences, and substance abuse), as well as inflammatory processes, can cause alterations in epigenetically regulated gene expression. In addition, epigenetic changes can contribute to the development and progression of certain diseases, such as periodontal disease, dental caries, congenital orofacial defects, and oral cancer. In summary, epigenetic modifications are potentially reversible and can be influenced by several lifestyle factors. Therefore, a deep understanding of these modifications will help to determine novel therapeutic targets in dental diseases, guided by appropriately indicated epigenetic biomarkers. Hopefully, these emerging biomarkers will allow the early detection, diagnosis, prognosis, and therapy of some common oral diseases.

\section{References}

1. Jośko-Ochojska J. Inheritance of trauma. Epigenetic "letter" to future generations. In: Medical and Social Aspects of Trauma. Katowice, Poland: Śląski Uniwersytet Medyczny; 2016:41-73.

2. Singh NN, Peer A, Nair S, Chaturvedi RK. Epigenetics: A possible answer to the undeciphered etiopathogenesis and behavior of oral lesions. J Oral Maxillofac Pathol. 2016;20(1):122-128.

3. Williams SD, Hughes TE, Adler CJ, Brook AH, Townsend GC. Epigenetics: A new frontier in dentistry. Aust Dent J. 2014;59(Suppl 1):23-33.

4. Seo JY, Park YJ, Yi YA, et al. Epigenetics: General characteristics and implications for oral health. Restor Dent Endod. 2015;40(1):14-22.

5. Packyanathan JS, Juneius CER. Role of epigenetic mechanisms in oral health: A review. Asian J Pharm. 2016;10(4):473-479.

6. Mohsin AHB, Barshaik S. Epigenetics in dentistry: A literature review. J Clin Epigenet. 2017;3:1. doi: 10.21767/2472-1158.100035

7. Barros SP, Offenbacher S. Modifiable risk factors in periodontal disease: Epigenetic regulation of gene expression in the inflammatory response. Periodontol 2000. 2014;64(1):95-110.

8. Ari G, Cherukuri S, Namasivayam A. Epigenetics and periodontitis: A contemporary review. JCDR. 2016;10(11):ZE07-ZE09. doi: 10.7860/ JCDR/2016/21025.8864

9. de Faria Amormino SA, Arão TC, Saraiva AM, et al. Hypermethylation and low transcription of TLR2 gene in chronic periodontitis. Hum Immunol. 2013;74(9):1231-1236.

10. Kowalski $\mathrm{P}$, Rubin $\mathrm{M}$, Kleer $\mathrm{C}$. E-cadherin expression in primary carcinomas of the breast and its distant metastases. Breast Cancer Res. 2003;5(6):R217-R222. doi: 10.1186/bcr651

11. Nagarakanti S. Differential expression of E-cadherin and cytokeratin 19 and net proliferative rate of gingival keratinocytes in oral epithelium in periodontal health and disease. J Periodontol. 2007;78(11): 2197-2202.

12. Loo WT, Jin L, Cheung MN, Wang M, Chow LW. Epigenetic change in E-cadherin and COX-2 to predict chronic periodontitis. J Trans/ Med. 2010;8:110. doi: 10.1186/1479-5876-8-110

13. Barnes PJ. Targeting the epigenome in the treatment of asthma and chronic obstructive pulmonary disease. Proc Am Thorac Soc. 2009;6 (8):693-696.

14. Zhang S, Barros SP, Niculescu MD, Moretti AJ, Preisser JS, Offenbacher S. Alteration of PTGS2 promoter methylation in chronic periodontitis. J Dent Res. 2010;89(2):133-137.

15. Lindroth AM, Park YJ. Epigenetic biomarkers: A step forward for understanding periodontitis. J Periodontal Implant Sci. 2013;43(3): 111-120.

16. Offenbacher S. Epigenetic regulation of TNFA expression in periodontal disease. J Periodontol. 2013;84(11):1606-1616.

17. Schulz S, Immel UD, Just L, Schaller HG, Gläser C, Reichert S. Epigenetic characteristics in inflammatory candidate genes in aggressive periodontitis. Hum Immunol. 2016;77(1):71-75.

18. Modesto A, Klein O, Tenuta LM, Gerlach RF, Vieira AR. Summary of the IADR cariology research, craniofacial biology, and mineralized tissue groups symposium, Iguaçu Falls, Brazil, June 2012: Gene-environment. Interactions and epigenetics in oral diseases: Enamel formation and its clinical impact on tooth defects, caries, and erosion. Dent 3000. 2013;1(1). http://dentistry3000.pitt.edu/ojs/index.php/ dentistry3000/article/view/16/17

19. Jośko-Ochojska J. Wpływ dramatycznych przeżyć i lęków matki ciężarnej na losy jej dziecka. In: Jośko-Ochojska J, ed. Lęk - nieodłaczny towarzysz człowieka od poczęcia aż do śmierci. Katowice, Poland: Śląski Uniwersytet Medyczny (SUM) in Katowice; 2013:11-35. 
20. Al-Jewair TS, Leake JL. The prevalence and risks of early childhood caries (ECC) in Toronto, Canada. J Contemp Dent Pract. 2010;11(5):1-8.

21. Hughes T, Bockmann M, Mihailidis S, et al. Genetic, epigenetic, and environmental influences on dentofacial structures and oral health: Ongoing studies of Australian twins and their families. Twin Res Hum Genet. 2013;16(1):43-51.

22. Apostolou $E$, Hochedlinger K. Chromatin dynamics during cellular reprogramming. Nature. 2013;502(7472):462-471.

23. Smith ZD, Meissner A. DNA methylation: Roles in mammalian development. Nat Rev Genet. 2013;14(3):204-220.

24. Delgado-Calle J, Sañudo C, Sanchez-Verde L, Garcia-Renedo RJ, Arozamena J, Riancho JA. Epigenetic regulation of alkaline phosphatase in human cells of the osteoblastic lineage. Bone. 2011;49(4): 830-838.

25. Delgado-Calle J, Sañudo C, Bolado A, et al. DNA methylation contributes to the regulation of sclerostin expression in human osteocytes. J Bone Miner Res. 2012;27(4):926-937.

26. Dawson MA, Kouzarides T. Cancer epigenetics: From mechanism to therapy. Cell. 2012;150(1):12-27.

27. Yoshioka H, Minamizaki T, Yoshiko Y.The dynamics of DNA methylation and hydroxymethylation during amelogenesis. Histochem Cell Biol. 2015;144(5):471-478.

28. Fernando S, Speicher DJ, Bakr MM, et al. Protocol for assessing maternal, environmental and epigenetic risk factors for dental caries in children. BMC Oral Health. 2015;15:167. doi: 10.1186/s12903-0150143-2

29. Hui T, Wang C, Chen D, Zheng L, Huang D, Ye L. Epigenetic regulation in dental pulp inflammation. Oral Dis. 2017;23(1):22-28.

30. Hui T, AP, Zhao Y, et al. EZH2, a potential regulator of dental pulp inflammation and regeneration. J Endod. 2014;40(8):1132-1138.

31. Huang GT, Garcia-Godoy F. Missing concepts in de novo pulp regeneration. J Dent Res. 2014;93(8):717-724.

32. Huang GT. Dental pulp and dentin tissue engineering and regeneration: Advancement and challenge. Front Biosci (Elite Ed). 2011;3: 788-800.

33. Duncan HF, Smith AJ, Fleming GJ, Cooper PR. Histone deacetylase inhibitors epigenetically promote reparative events in primary dental pulp cells. Exp Cell Res. 2013;319(10):1534-1543.

34. Qiu X, Xiao X, Li N, Li Y. Histone deacetylases inhibitors (HDACis) as novel therapeutic application in various clinical diseases. Prog Neuropsychopharmacol Biol Psychiatry. 2017;72:60-72.

35. Paino F, La Noce $M$, Tirino $V$, et al. Histone deacetylase inhibition with valproic acid downregulates osteocalcin gene expression in human dental pulp stem cells and osteoblasts: Evidence for HDAC2 involvement. Stem Cells. 2014;32(1):279-289.

36. Wyszynski DF. Cleft Lip and Palate: From Origin to Treatment. New York, NY: Oxford University Press (US); 2002.

37. Plamondon JA, Harris MJ, Mager DL, Gagnier L, Juriloff DM. The clf2 gene has an epigenetic role in the multifactorial etiology of cleft lip and palate in the A/WySn mouse strain. Birth Defects Res A Clin Mol Teratol. 2011;91(8):716-727.

38. Little J, Cardy A, Munger RG. Tobacco smoking and oral clefts: A meta-analysis. Bull World Health Organ. 2004;82(3):213-218.

39. Grosen D, Petersen B, Skytthe A, et al. Risk of oral clefts in twins. Epidemiology. 2011;22(3):313-319.

40. Kuriyama M, Udagawa A, Yoshimoto S, et al. DNA methylation changes during cleft palate formation induced by retinoic acid in mice. Cleft Palate Craniofac J. 2008;45(5):545-551.

41. Conrad R, Barrier M, Ford LP. Role of miRNA and miRNA processing factors in development and disease. Birth Defects Res CEmbryo Today. 2006;78(2):107-117.
42. Seelan RS, Mukhopadhyay P, Pisano MM, Greene RM. Developmental epigenetics of the murine secondary palate. ILAR J. 2012;53(3-4): 240-252.

43. Warner DR, Bhattacherjee V, Yin X, et al. Functional interaction between Smad, CREB binding protein, and p68 RNA helicase. Biochem Biophys Res Commun. 2004;324(1):70-76.

44. Ornoy A. Valproic acid in pregnancy: How much are we endangering the embryo and fetus? Reprod Toxicol. 2009;28(1):1-10.

45. Pandorf CE, Haddad F, Wright C, Bodell PW, Baldwin KM. Differential epigenetic modifications of histones at the myosin heavy chain genes in fast and slow skeletal muscle fibers and in response to muscle unloading. Am J Physiol Cell Physiol. 2009;297(1):6-16.

46. Desh H, Gray SL, Horton MJ, et al. Molecular motor MYO1C, acetyltransferase KAT6B and osteogenetic transcription factor RUNX2 expression in human masseter muscle contributes to development of malocclusion. Arch Oral Biol. 2014;59(6):601-607.

47. Rowlerson A, Raoul G, Daniel Y, et al. Fiber-type differences in masseter muscle associated with different facial morphologies. Am JOrthod Dentofacial Orthop. 2005;127(1):37-46.

48. Baar K. Epigenetic control of skeletal muscle fibre type. Acta Physiol (Oxf). 2010;199(4):477-487.

49. Deng P, Chen QM, Hong C, Wang CY. Histone methyltransferases and demethylases: Regulators in balancing osteogenic and adipogenic differentiation of mesenchymal stem cells. Int J Oral Sci. 2015;7(4): 197-204.

50. Huh A, Horton MJ, Cuenco KT, et al. Epigenetic influence of KAT6B and HDAC4 in the development of skeletal malocclusion. Am JOrthod Dentofacial Orthop.2013; 144(4):568-576.

51. Cho $\mathrm{Y}, \mathrm{Kim} B, \mathrm{Bae} H$, et al. G. Direct gingival fibroblast/osteoblast transdifferentiation via epigenetics. J Dent Res. 2017;96(5):555-561. doi: 10.1177/0022034516686745

52. Guttal KS, Naikmasur VG, Bhargava P, Bathi RJ. Frequency of developmental dental anomalies in the Indian population. Eur J Dent. 2010;4(3):263-269.

53. Wang J, Sun K, Shen Y, et al. DNA methylation is critical for tooth agenesis: Implications for sporadic non-syndromic anodontia and hypodontia. Sci Rep. 2016;6:19162. doi: 10.1038/srep19162

54. Townsend G, Richards L, Hughes T, Pinkerton S, Schwerdt W. Epigenetic influences may explain dental differences in monozygotic twin pairs. Aust Dent J. 2005;50(2):95-100.

55. Suda N, Hattori M, Kosaki K, et al. Correlation between genotype and supernumerary tooth formation in cleidocranial dysplasia. Orthod Craniofac Res. 2010;13(4):197-202.

56. Brook AH. Multilevel complex interactions between genetic, epigenetic and environmental factors in the etiology of anomalies of dental development. Arch Oral Biol. 2009;54(Suppl 1):3-17.

57. Langevin SM, Butler RA, Eliot $M$, et al. Novel DNA methylation targets in oral rinse samples predict survival of patients with oral squamous cell carcinoma. Oral Oncol. 2014;50(11):1072-1080.

58. Ribeiro IP, Caramelo F, Marques F, et al. WT1, MSH6, GATA5 and PAX5 as epigenetic oral squamous cell carcinoma biomarkers - a short report. Cell Oncol (Dordr). 2016;39(6):573-582.

59. Sushma PS, Jamil K, Kumar PU, Satyanarayana U, Ramakrishna M, Triveni B. PTEN and $p 16$ genes as epigenetic biomarkers in oral squamous cell carcinoma (OSCC): A study on south Indian population. Tumour Biol. 2016;37(6):7625-7632.

60. Cheng JC, Chiang MT, Lee $\mathrm{CH}$, et al. $\gamma$-Synuclein expression is a malignant index in oral squamous cell carcinoma. J Dent Res. 2016;95(4): 439-445. 